\title{
Editorial
}

\section{Compulsive Internet Use: Is Addiction Medicine Concerned?}

\author{
Daniele Fabio Zullino* and Yasser Khazaal
}

\author{
Division of Addictology, University Hospitals of Geneva, Switzerland
}

\section{COMPULSIVE INTERNET USE: IS ADDICTION MEDICINE CONCERNED?}

Besides many other technological advances, the last two decennia have particularly been characterized by the appearance and rapid expansion of internet, its innovations and its countless products. On one side, its rapid and often overwhelming imposition into many aspects of our daily life is becoming an increasing challenge for many people. On the other side, there is a growing evidence of excessive utilization patterns among a considerable part of internet users, which came to the attention of mental health professionals in many countries over the world.

Internet addiction as a diagnosis was in the beginning proposed by Ivan Goldberg in a satirical hoax on PsyCom.Net, but has then rapidly been adopted by many clinicians and researchers. There has since been a sometimes passionate debate about the validity of the concept of internet addiction, positions ranging from sometimes apocalyptic visions to those declaring the proposed diagnostic category a pure attempt of disease mongering.

The question of defining a new diagnosis is of great importance, as it can deeply affect scientific research, access to resources, and treatment selection in clinical practice.

One of the main rational for proposing internet addiction as a diagnosis on its own is the perceived need among most professionals working in the field to increase the coverage of the system.

A new diagnosis is usually intended to describe a pattern of symptoms, which is not already captured in the existing categories [1]. A new diagnosis can be incorporated into an existing system in at least 3 ways. It can (a) be suggested to constitute a new category not already present in the current diagnostic system (e.g. a manual like DSM or ICD), or (b) to be a new category similar to currently existing categories but subthreshold in its requirements, or (c) be advocated to represent a specific subtype of an existing disorder with claimed clinical (e.g. therapeutic) implications. The last is mostly proposed to be the case regarding internet addiction. The papers of the present special edition of the TOADDJ center on this assumption, i.e. that internet addiction are a sub-category of addictive behaviors.

*Address correspondence to this author at the Division of Addictology, University Hospitals of Geneva, Grand Pré 70C, CH - 1202 - Genève, Switzerland; Tel: + 412237255 50; Fax: + 412232817 60;

E-mail: Daniele.Zullino@hcuge.ch
As the links between neurobiological processes and behavior are, like in other psychiatric domains, highly complex, the related research remains particularly dependent on the principles and process of syndrome identification. When defining a subtype of a diagnostic category, as it is the case for internet addiction, finding similarities between the new entity (internet addiction) and those already established (e.g. cocaine addiction) may be a momentous element to corroborate the claim.

The present article series is aimed to contribute to this debate. The approach chosen is to review current practices in the field of internet addiction and to compare them with practices within widely accepted field of addiction medicine.

The paper by Thorens et al. reviews available data on certain features of internet applications which can be thought of acting on the same neurobiological structures involved in substance-related addictions, e.g. the mesolimbic dopaminergic system. It will be argued that many internet applications (e.g. MMORPG, pornography etc.) are particularly well suited to activate this pathway.

Billieux et al., argue in their paper that, to be considered as an addiction, internet addiction has to share at least some of the central characteristics with other, well accepted, addictions. They discuss poor self-regulation capacities as such a common factor.

What are the current psychotherapeutic options? Psychotherapeutic interventions developed for internet addiction have mainly been derived from other addictions. The paper by Khazaal et al., review the approaches proposed until now, and comment on the provisional efficacy data.

What are the current pharmacotherapeutic promises? In this final paper, Achab et al., review the available observations on pharmacotherapy and propose an outlook on the most promising pharmacotherapeutic scenarios.

Paralleling, thus, established practices in addiction medicine with those related to this emerging clinical problem can hopefully contribute to the discussion about a potential place of technology addictions within the spectrum of addictive disorders.

Finally, it should, however, be kept in mind that "Internet addiction" may be a somewhat imprecise term as it leads to muddle-up the addictive product (specific applications, e.g. pornography) with the vector (internet technology). The same, still, could be said for different other addictions. The same is e.g. true for nicotine (the addictive product) and the 
cigarette (the vector). The same vector (cigarette) can carry different addictive products (nicotine, cannabis etc.).

Thus, we probably should rather think about diverse addictions, which developments are all sustained by a same medium: internet.

\section{ACKNOWLEDGEMENT}

Declared none.

\section{CONFLICT OF INTEREST}

Declared none.

\section{REFERENCE}

[1] Pincus HA, Frances A, Davis WW, First MB, Widiger TA. DSMIV and new diagnostic categories: holding the line on proliferation. Am J Psychiatry 1992; 149(1): 112-7.

(C) Zullino and Khazaal; Licensee Bentham Open.

This is an open access article licensed under the terms of the Creative Commons Attribution Non-Commercial License (http://creativecommons.org/licenses/ by-nc/3.0/) which permits unrestricted, non-commercial use, distribution and reproduction in any medium, provided the work is properly cited. 